\title{
Some remarks on the thermodynamics of charged AdS black holes with cloud of strings and quintessence
}

\author{
J. M. Toledo ${ }^{\mathrm{a}}$, V. B. Bezerra ${ }^{\mathrm{b}}$ \\ Departamento de Física, Universidade Federal daParaíba, Caixa Postal 5008, João Pessoa, PB, Brazil
}

Received: 26 September 2018 / Accepted: 22 January 2019 / Published online: 4 February 2019

(C) The Author(s) 2019

\begin{abstract}
In this paper we investigate the combined effects of the cloud of strings and quintessence which surround a Reissner-Nordstrom AdS black hole on its thermodynamics. We treat the cosmological constant as a thermodynamic pressure whose conjugate variable is the volume. It is shown how the cloud of strings affect the thermodynamic variables, as for example, temperature, entropy, internal energy and heat capacity. The critical expressions for temperature, pressure and specific volume are obtained and the critical exponents calculated. Concerning the heat engine, we calculate its efficiency and show how this quantity is influenced by the presence of the cloud of strings and quintessence.
\end{abstract}

\section{Introduction}

Studies concerning a formalism to obtain the solutions of the Einstein equations corresponding to a black hole surrounded by a cloud of strings were initiated by Letelier [1]. These studies were motivated, originally, by the fact that the nature can be represented, in principle, by a collection of extended objects, like one-dimensional strings, rather than of point particles, in a more appropriate way.Among various results obtained by Letelier [1], as for example, for plane, cylindrical and spherical symmetries, the one of our interest concerns the spherical symmetry and corresponds to a generalization of the Schwarzschild solution, more specifically, corresponds to the Schwarzschild black hole surrounded by a spherically symmetric cloud of strings [1]. In other words, it corresponds to a modification of Schwarzschild solution by taking a cloud of strings as an additional source of the gravitational field. Concerning this solution, if we consider the mass of the central body (black hole) equal to zero, we have only a cloud of strings, in which case we have no horizon, but just a naked singularity at $r=0$. Returning to

\footnotetext{
${ }^{a}$ e-mail: jefferson.m.toledo@gmail.com

be-mail: valdir@fisica.ufpb.br
}

the combined sources, black hole and cloud of strings, we can conclude that the presence of the cloud of strings produces an effect of global origin associated with a solid deficit angle which depends on the parameter that codifies the presence of the cloud, similarly to what happens in the spacetime of a global monopole [2]. Additionally, the presence of the cloud produces a profound influence on the horizon structure, enlarging the radius of the horizon event as compared with the Schwarzschild radius. Thus, the effects of global origin associated with the solid deficit angle, which is determined by the presence of the cloud of strings, will produce possible astrophysical consequences, which justify the investigation of the role played by the cloud of strings in this scenario.

The astronomical observations confirming that the universe experiences an accelerated expansion constitutes one of the most important discoveries of the era of the observational cosmology [3]. This fact implies that at large scale structure, there exists a gravitationally repulsive energy component which indicates the existence of a negative pressure. One of the possible origins of this negative pressure is associated to a fluid that permeates the universe, with energy density constant in time and spatially uniform. In this case, the pressure is proportional to the energy density, with the proportionality constant assuming values in the interval $(-1$, $-1 / 3)$, in order to have the desired cosmic acceleration. This kind of scenario corresponds to what is called quintessence or quintessence dark energy [4]. This permeates the universe and consequently surrounds a black hole and certainly produces profound astrophysical consequences. Therefore, it is important to know the solution of the Einstein equations for black holes surrounded by quintessence as was done by Kiselev [4].

The black hole surrounded by quintessence, in the way obtained by Kiselev [4] has been extensively studied in the literature even though this solution is not free of problems that deserve further discussion. We can cite, for example, the 
correspondence of the Keselev stress-energy tensor with the scalar model for quintessence and the fact that, if we consider the black hole-free solution, the metric is not homogeneous, as expected for cosmological models.

Black hole thermodynamics is one of the topics in gravitational physics which becomes an active and important area of research since the works of Bekenstein [5,6] and Hawking [7-9] in the 1970's up to now, in part, due to the window it opens to understand the connection between quantum mechanics and gravity, providing a deep insight in which concerns the understanding of the nature of quantum gravity.

The study of the thermodynamical features of charged AdS black holes showed that there exists a similarity between these astrophysical objects and liquid-gas systems. In particular, Reissner-Nordstrom AdS black holes present firstorder phase transitions whose critical behavior is similar to the ones experienced by a system in condensed matter $[10,11]$. Investigations concerning this topic have enhanced after the suggestion that the cosmological constant should be treated as the thermodynamic variable [12-16], which is interpreted as the thermodynamic pressure with the thermodynamic volume being the conjugate quantity. Taking this fact into account, more attention has been done to the study of black hole thermodynamics of AdS black holes [15-28], especially with respect to charged AdS black holes by the reason that there is an analogy between these objects and liquid-gas systems, phase transitions appear [17] and these must be investigated in order to find the whole phase structure, through the determination of the critical points associated with the thermodynamical quantities, but now, taking into account not only the quintessence, but also the presence of a cloud of strings.

Another idea that appears in the framework of black hole thermodynamics is related to the heat engine [29]. In this case, the efficiency can be determined analogously to a traditional heat engine [29-33]. Taking the pressure and the volume as dynamical variables, it is possible to extract mechanical work by using the $p d V$ term. Similarly to what happens in classical thermodynamics, a heat engine is defined by a closed cycle in the $\mathrm{pV}$ plane [29]. In this scenario, the efficiency of the heat engines has been analyzed in different black hole spacetimes [26,29,34], among others.

In this paper, we analyze the thermodynamics of a Reissner-Nordstrom AdS black hole surrounded by a cloud of strings and quintessence, by calculating different thermodynamic variables, determining their critical values as well as the efficiency of a heat engine.

It is already known that a cloud of strings decreases the real and imaginary parts of the quasinormal frequencies of a black hole surrounded by quintessence, approaching the system from the astrophysical scenario [35].

In the present work, it will be shown that the presence of the cloud of strings gives rise to important issues in the ther- modynamics of the black hole, if we compare with the one surrounded only by quintessence [36]. The cloud of strings parameter decreases the thermodynamic quantities as temperature, entropy and internal energy of the black hole. Furthermore, it increases the efficiency of the black hole considered as a heat engine. These important effects are extensively discussed in this paper.

This paper is organized as follows. In Sect. 2, we present the metric and the event horizons. In Sect. 3, we discuss the thermodynamics. In Sect. 4, the critical values for some thermodynamic variables and critical exponents are determined. In Sect. 5, we discuss how the black hole considered can be treated as a heat engine. Finally, in Sect. 6, we present the concluding remarks.

\section{The metric and event horizons}

In this section, we obtain the metric corresponding to the spacetime generated by a charged static black hole with cosmological constant and surrounded by a cloud of strings and quintessence.

Recently, it was obtained the solution corresponding to a black hole immersed in quintessence, whose energymomentum tensor is given by [4]

$T_{t}^{t}=T_{r}^{r}=\rho_{q}$,

$T_{\theta}^{\theta}=T_{\phi}^{\phi}=-\frac{1}{2} \rho_{q}\left(3 \omega_{q}+1\right)$,

where the pressure and density of quintessence are related by the equation of state $p_{q}=\omega_{q} \rho_{q}$, with $\omega_{q}$ being the quintessential state parameter.

If we consider this mass-energy content, we can adopt the static spherically symmetric metric background given

$d s^{2}=f(r) d t^{2}-\frac{1}{f(r)} d r^{2}-r^{2} d \Omega^{2}$.

with [4]

$f(r)=1-\frac{2 M}{r}-\frac{\alpha}{r^{3 \omega_{q}+1}}$,

where $\alpha$ and $M$ are normalization constants, which should be related to the quintessence and mass, respectively. With this solution, the spacetime metric is given by

$$
\begin{aligned}
d s^{2}= & \left(1-\frac{2 M}{r}-\frac{\alpha}{r^{3 \omega_{q}+1}}\right) d t^{2} \\
& -\left(1-\frac{2 M}{r}-\frac{\alpha}{r^{3 \omega_{q}+1}}\right)^{-1} d r^{2}-r^{2} d \Omega^{2}
\end{aligned}
$$


which corresponds to a static black hole, with mass $M$, surrounded by quintessence, with density $\rho_{q}$ given by

$\rho_{q}=-\frac{\alpha}{2} \frac{3 \omega_{q}}{r^{3\left(\omega_{q}+1\right)}}$.

In order to get the scenario of accelerated expansion, it is necessary to impose that $-1<\omega_{q}<-1 / 3$. As to $\alpha$, it is a positive parameter such that, when it is equal to zero, we recover the Schwarzschild solution.

The metric given by Eq. (5) can be generalized by taking into account the presence of an electromagnetic field due to a charge $Q$, as well as, the cosmological constant. In this case, Eq. (5) turns into [4,26,36,37]

$$
\begin{aligned}
d s^{2}= & \left(1-\frac{2 M}{r}+\frac{Q^{2}}{r^{2}}-\frac{\alpha}{r^{3 \omega_{q}+1}}-\frac{\Lambda r^{2}}{3}\right) d t^{2} \\
& -\left(1-\frac{2 M}{r}+\frac{Q^{2}}{r^{2}}-\frac{\alpha}{r^{3 \omega_{q}+1}}-\frac{\Lambda r^{2}}{3}\right)^{-1} d r^{2} \\
& -r^{2} d \Omega^{2} .
\end{aligned}
$$

The solution of Einstein's equations describing a black hole with a cloud of strings was obtained by Letelier [1]. First of all, we can consider that a moving infinitesimally thin string traces out a two-dimensional world sheet, $\Sigma$, which can be described by the equation

$x^{\mu}=x^{\mu}\left(\xi^{a}\right), \quad a=0,1$,

with $\xi^{0}$ and $\xi^{1}$ being timelike and spacelike parameters, respectively. Thus, the string is characterized by its world sheet. The induced metric, $h_{a b}$, on the world sheet is given by

$h_{a b}=g_{\mu \nu} \frac{\partial x^{\mu}}{\partial \xi^{a}} \frac{\partial x^{\nu}}{\partial \xi^{b}}$.

It can be associated to the string world sheet, a bivector $\Sigma^{\mu \nu}$, such that $[1]$

$\Sigma^{\mu \nu}=\epsilon^{a b} \frac{\partial x^{\mu}}{\partial \xi^{a}} \frac{\partial x^{v}}{\partial \xi^{b}}$

where $\epsilon^{a b}$ is the two-dimensional Levi-Civita symbol, with $\epsilon^{01}=-\epsilon^{10}=1$.

Now, let us consider the energy-momentum tensor for a cloud of strings, characterized by a proper density $\rho$, which can be written as [1]

$T^{\mu \nu}=\frac{\rho \Sigma^{\mu \beta} \Sigma_{\beta}{ }^{\nu}}{\sqrt{-h}}$,

where $h=\frac{1}{2} \Sigma^{\mu \nu} \Sigma_{\mu \nu}$ is the determinant of the induced metric.
As the string cloud is spherically symmetric, the density, $\rho$, and the bivector $\Sigma^{\mu \nu}$, should be functions only of the radial coordinate. In this case, the non vanishing components of the bivector are $\Sigma^{01}$ and $\Sigma^{10}$, which are related by $\Sigma^{01}=$ $-\Sigma^{10}$. Thus, $T_{0}^{0}=T_{1}^{1}=-\rho \Sigma^{01}$ and, using the relation [1]

$\frac{d}{d r}\left[\left(r^{2} T_{0}^{0}\right)^{\frac{1}{2}}\right]=0$,

we find the following result

$T_{0}^{0}=T_{1}^{1}=\frac{a}{r^{2}}$,

$T_{2}^{2}=T_{3}^{3}=0$,

where $a$ is an integration constant which is related to the presence of the cloud of strings. Using this mass-energy configuration, Letelier determined the spacetime metric of a black hole surrounded by a cloud of strings, which is written as [1]

$$
\begin{aligned}
d s^{2}= & \left(1-a-\frac{2 M}{r}\right) d t^{2}-\left(1-a-\frac{2 M}{r}\right)^{-1} d r^{2} \\
& -r^{2} d \Omega^{2} .
\end{aligned}
$$

Now, let us assume that the quintessence and the cloud of strings do not interact with each other, such that we can consider the energy-momentum tensor for both sources as a linear superposition given as follows

$T_{t}^{t}=T_{r}^{r}=\rho_{q}+\frac{a}{r^{2}}$,

$T_{\theta}^{\theta}=T_{\phi}^{\phi}=-\frac{1}{2} \rho_{q}\left(3 \omega_{q}+1\right)$.

Considering, once again, the static spherically symmetric line element given by Eq. (3), we get the line element associated with a charged black hole surrounded by a cloud of strings and quintessence which is written as

$$
\begin{aligned}
d s^{2}= & \left(1-a-\frac{2 M}{r}+\frac{Q^{2}}{r^{2}}-\frac{\alpha}{r^{3 \omega_{q}+1}}-\frac{\Lambda r^{2}}{3}\right) d t^{2} \\
& -\left(1-a-\frac{2 M}{r}+\frac{Q^{2}}{r^{2}}-\frac{\alpha}{r^{3 \omega_{q}+1}}-\frac{\Lambda r^{2}}{3}\right)^{-1} d r^{2} \\
& -r^{2} d \Omega^{2} .
\end{aligned}
$$

In what follows, we will fix $\omega_{q}=-2 / 3$.

Now, let us analyze the existence of horizons which are determined by imposing that

$f(r)=1-a-\frac{2 M}{r}+\frac{Q^{2}}{r^{2}}-\alpha r-\frac{\Lambda r^{2}}{3}=0$.

The black hole has three positive horizons: the black hole event horizon, $r_{+}$, the internal (Cauchy) horizon, $r_{-}$, and a quintessential cosmological horizon, $r_{q}$.

In Fig. 1, the graphs of the function $f(r)$ is shown for different values of the parameters $a$ and $\alpha$, which codify 


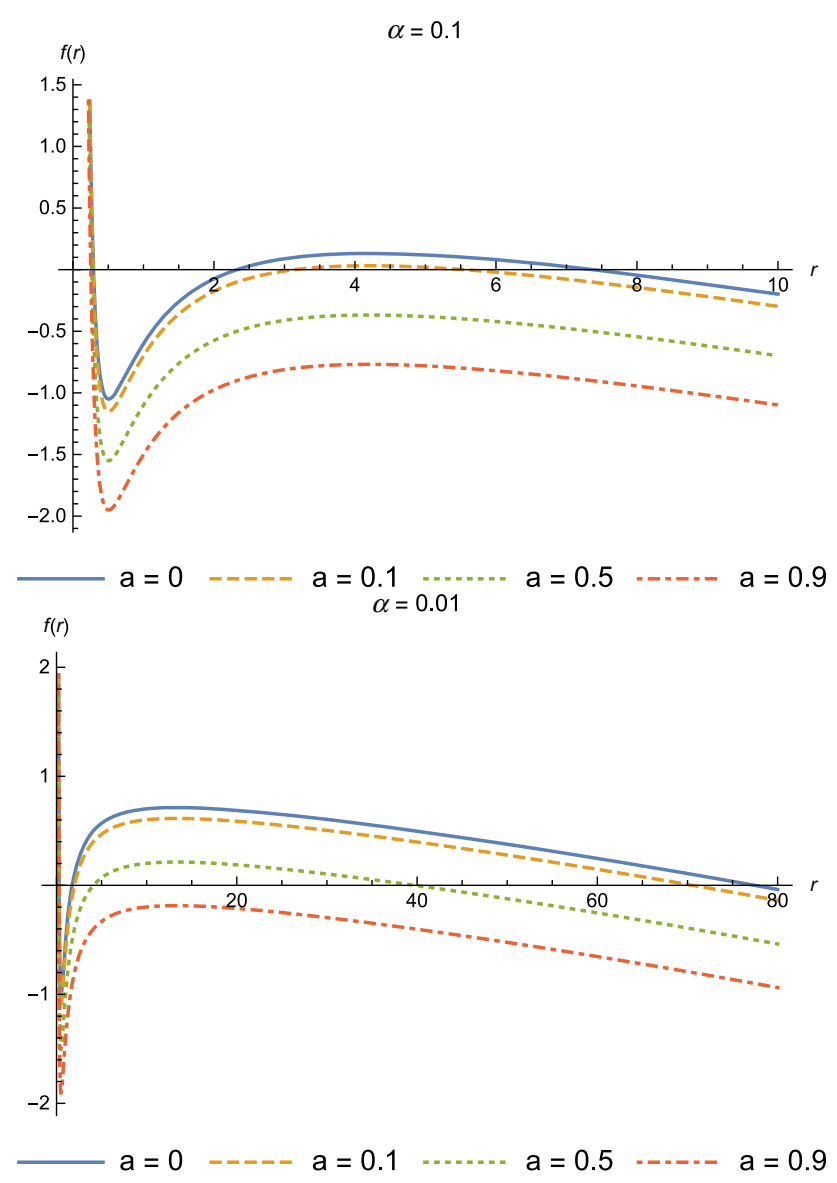

Fig. 1 The function $f(r)$ for different values of $a$ and $\alpha$. We choose $M=1$ and $Q^{2}=0.5$

the presence of the cloud of strings and the quintessence, respectively.

\section{Black hole thermodynamics}

In this section, we investigate the black hole thermodynamics, analyzing the thermodynamic potentials and intensive parameters.

For any horizon radius $r_{h}$, the mass parameter of the black hole surrounded by a cloud of strings and quintessence can be written as

$M=\frac{1-a}{2} r_{h}+\frac{Q^{2}}{2 r_{h}}-\frac{\alpha}{2} r_{h}^{2}-\frac{\Lambda r_{h}^{3}}{6}$.

For a static spherically symmetric black hole, the entropy can be calculated by the area law, which is written as $[5,6]$
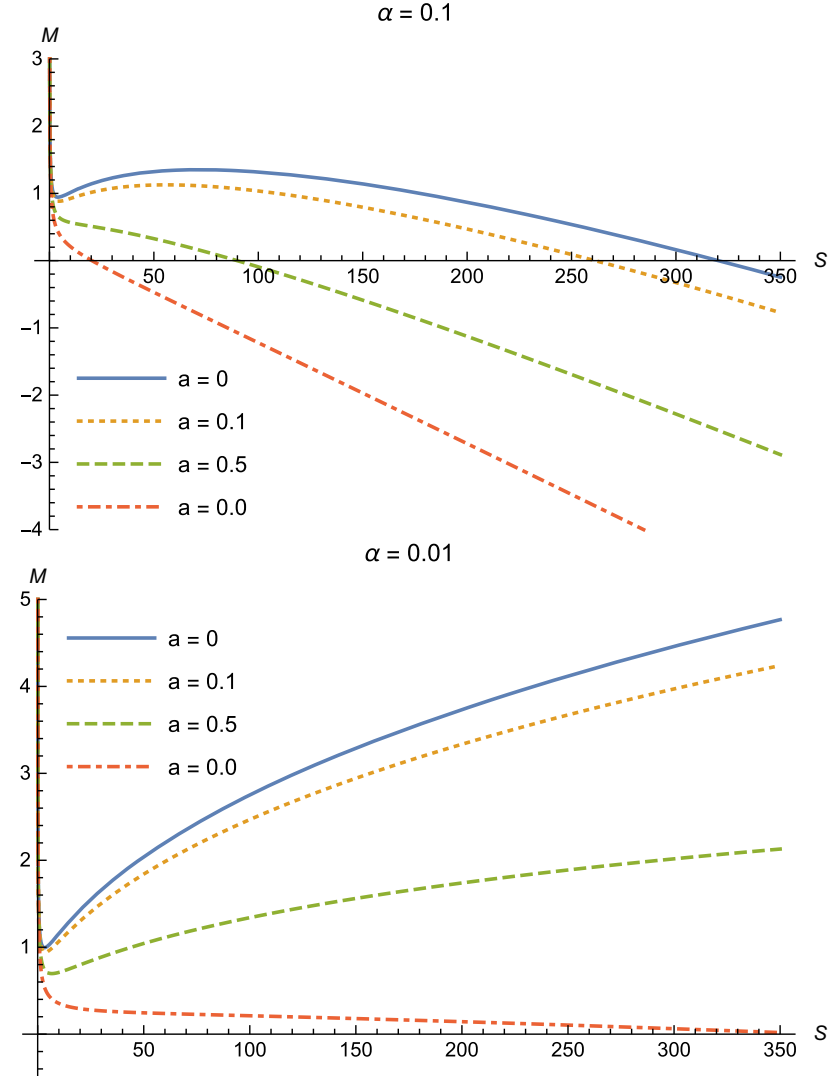

Fig. 2 Mass parameter as a function of the entropy, $S$, for different values of $a$ and $\alpha$

$S=\frac{A}{4}=\pi r_{h}^{2}$,

and thus, it is a monotonic function of the horizon radius.

Therefore, as a function of the black hole entropy, the mass parameter, which corresponds to the mass (energy) of the charged black hole modified due to the presence of the cosmological constant, cloud of strings and quintessence, is given by

$M=M(S, Q, \Lambda)=\frac{1-a}{2} \sqrt{\frac{S}{\pi}}+\frac{Q^{2}}{2} \sqrt{\frac{\pi}{S}}-\frac{\alpha}{2} \frac{S}{\pi}-\frac{\Lambda}{6}\left(\frac{S}{\pi}\right)^{3 / 2}$

and its behavior is represented in Fig. 2 for different values of the cloud of string and quintessence parameters.

Now, let us assume that in the context of the black hole thermodynamics, the pressure is related to the cosmological constant by [16]

$p=-\frac{\Lambda}{8 \pi}$.

Thus, the mass parameter turns into 


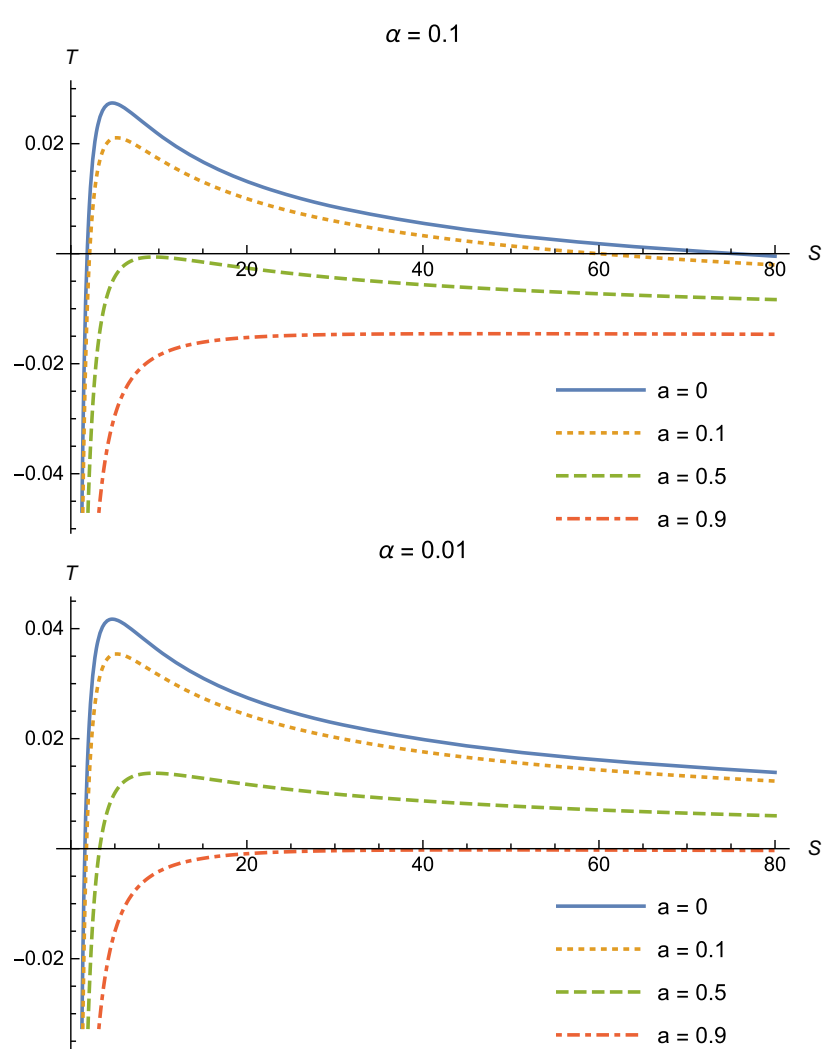

Fig. 3 The Hawking temperature $T$ as a function of the entropy

$$
\begin{aligned}
M= & M(S, Q, p)=\frac{1-a}{2} \sqrt{\frac{S}{\pi}}+\frac{Q^{2}}{2} \sqrt{\frac{\pi}{S}}-\frac{\alpha}{2} \frac{S}{\pi} \\
& +\frac{4 \pi p}{3}\left(\frac{S}{\pi}\right)^{3 / 2} .
\end{aligned}
$$

Due to its functional dependence, the mass parameter $M=M(S, Q, p)$ can be associated with the enthalpy $H=H(S, Q, p)$. Thus, the first law of black hole thermodynamics is written as

$\delta M=T \delta S+\phi d Q+V \delta p$,

where $T$ is the Hawking temperature [8,9], whose behavior as a function of entropy is shown in Fig. 3, and is given by

$T=\left(\frac{\partial M}{\partial S}\right)_{Q, p}=\frac{1-a}{4 \sqrt{S \pi}}-\frac{Q^{2}}{4 S} \sqrt{\frac{\pi}{S}}-\frac{\alpha}{2 \pi}+2 p \sqrt{\frac{S}{\pi}}$.

In which concerns the electric potential $\phi$ and the volume parameter $V$, they are obtained by

$$
\begin{aligned}
& \phi=\left(\frac{\partial M}{\partial Q}\right)_{S, p}=Q \sqrt{\frac{\pi}{S}}=\frac{Q}{r_{h}}, \\
& V=\left(\frac{\partial M}{\partial p}\right)_{S, Q}=\frac{4 \pi}{3}\left(\frac{S}{\pi}\right)^{3 / 2}=\frac{4 \pi r_{h}^{3}}{3} .
\end{aligned}
$$

As should be expected, the thermodynamical volume is a monotonic function of the horizon radius, given by $4 \pi r_{h}^{3} / 3$ and the electric potential is the one studied in classical electromagnetism. Note that, from Eqs. (21) and (28), we conclude that the entropy and the thermodynamical volume are not independent.

The internal energy of the black hole is obtained from Eq. (24) by a Legendre transformation and, thus, we have

$$
\begin{aligned}
E(S, Q, V) & =M(S, Q, p)-V p \\
& =\frac{1-a}{2} \sqrt{\frac{S}{\pi}}+\frac{Q^{2}}{2} \sqrt{\frac{\pi}{S}}-\frac{\alpha}{2} \frac{S}{\pi}
\end{aligned}
$$

On the other hand, the black hole heat capacity at constant pressure is calculated using the relation between entropy and the temperature obtained from Eq. (26), and thus we have

$$
\begin{aligned}
C_{p} & =\left.T \frac{\partial S}{\partial T}\right|_{Q, p} \\
& =\frac{2(1-a) S^{2}-2 Q^{2} S-4 \alpha S^{2} \sqrt{\frac{S}{\pi}}+16 p S^{3}}{-(1-a) S+3 Q^{2}+8 p S^{2}},
\end{aligned}
$$

while the heat capacity at constant volume is given by

$C_{V}=\left.T \frac{\partial S}{\partial T}\right|_{Q, V}=0$

Thus, we can conclude that $C_{V}$ vanishes since, from Eq. (28), one can verify that, if $V$ is constant, $S$ is also constant, which means that, for this black hole, isochoric processes are also adiabatic ones, due to the relations between the entropy and the volume.

The behavior of $C_{p}$ as a function of entropy is shown in Fig. 4. Note that there are second-order phase transitions for different values of the parameters $\alpha$ and $a$, except for $a=0.9$, in which case this transition is suppressed.

If we rewrite Eq. (26) as a function of $p$ and $V$, we find the equation of state

$T(p, V)=\frac{1-a}{(4 \pi)^{2 / 3}(3 V)^{1 / 3}}-\frac{Q^{2}}{3 V}-\frac{\alpha}{2 \pi}+2 p\left(\frac{3 V}{4 \pi}\right)^{1 / 3}$.

whose diagrams $p \times V$ are shown in Figs. 5, 6 and 7 .

Finally, let us consider that the parameters related to the cloud of string and the quintessence, respectively $a$ and $\alpha$, are extensive thermodynamic parameters [26]. As a consequence, the first law of thermodynamics must be modified to

$\delta M=T \delta S+\phi \delta Q+V \delta p+\mathscr{A} \delta \alpha+\mathscr{B} \delta a$.

with

$\mathscr{A}=-\frac{r_{h}^{2}}{2}=-\frac{S}{2 \pi}$ 

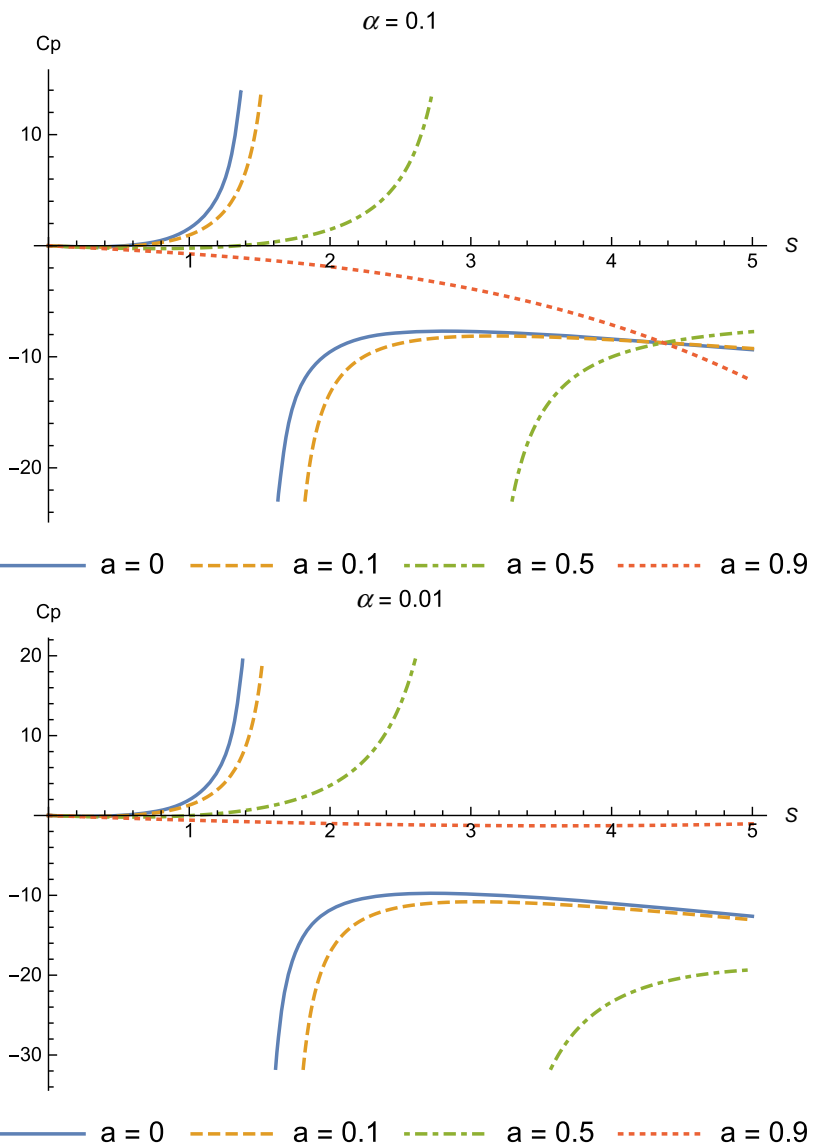

Fig. 4 The heat capacity at constant pressure, $C_{p}$, as a function of the entropy, $S$

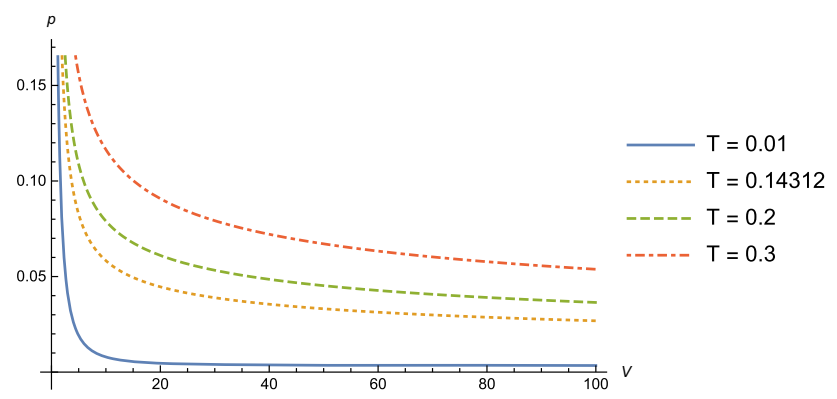

Fig. 5 Graphs $p \times V$ for different values of $T$. We assumed that $Q=$ $M=1$ e $a=0.1$

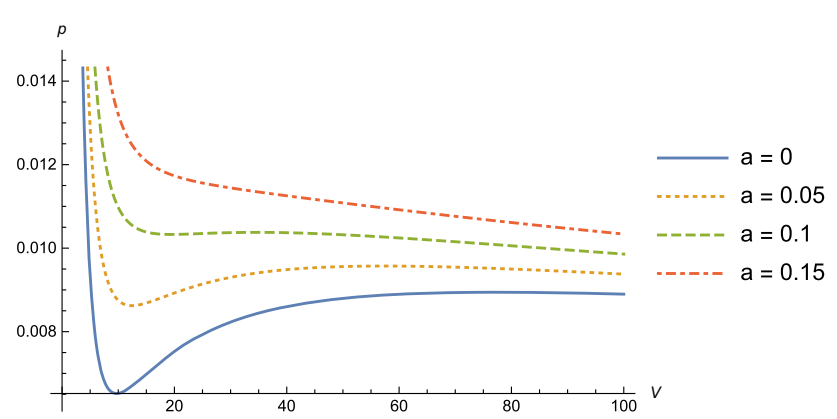

Fig. 6 Graphs $p \times V$ for $T=0.1, \alpha=0.01$ and different values of $a$

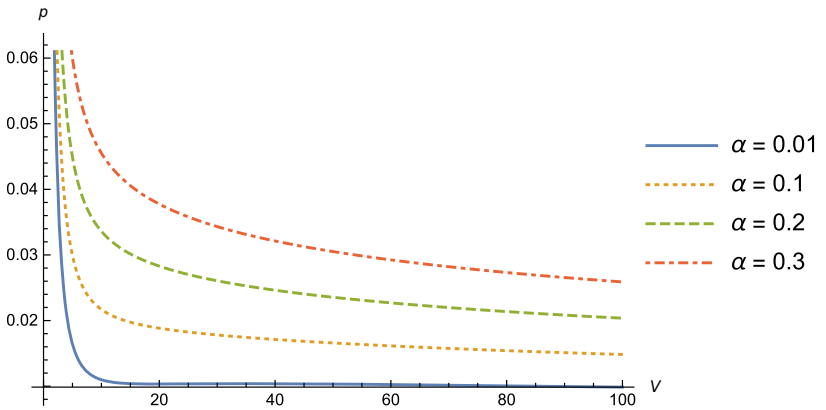

Fig. 7 Graphs $p \times V$ for $T=0.1, a=0.1$ and different values of $\alpha$

and

$\mathscr{B}=-\frac{r_{h}}{2}=-\frac{1}{2} \sqrt{\frac{S}{\pi}}$,

where $\mathscr{A}$ and $\mathscr{B}$ are conjugate to the parameters $\alpha$ and $a$, respectively.

\section{Critical thermodynamic variables}

In this section, let us investigate the critical thermodynamic variables and determine the critical exponents.

The Hawking temperature given by Eq. (26) can be written as a function of $r_{h}$ as

$T=\frac{1-a}{4 \pi r_{h}}-\frac{Q^{2}}{4 \pi r_{h}^{3}}-\frac{\alpha}{2 \pi}+2 p r_{h}$,

from which we can obtain the equation of state as

$p=\frac{T}{2 r_{h}}-\frac{1-a}{8 \pi r_{h}^{2}}+\frac{Q^{2}}{8 \pi r_{h}^{4}}+\frac{\alpha}{4 \pi r_{h}}$.

Now let us define $v=2 r_{h}$ [36], which is the double of the horizon radius and differs from the usual definition of specific volume in thermodynamics. Thus, Eq. (37) turns into

$p=\frac{T}{v}-\frac{1-a}{2 \pi v^{2}}+\frac{2 Q^{2}}{\pi v^{4}}+\frac{\alpha}{2 \pi v}$.

In order to obtain the critical points, let us take into account the following conditions

$\left.\frac{\partial p}{\partial v}\right|_{T=T_{c}}=0$

$\left.\frac{\partial^{2} p}{\partial v^{2}}\right|_{T=T_{c}}=0$.

Using the first condition, we get the following relation between the critical Hawking temperature, $T_{c}$, and the critical 
specific volume, $v_{c}$,

$T_{c}=\frac{1-a}{\pi v_{c}}-\frac{8 Q^{2}}{\pi v_{c}^{3}}-\frac{\alpha}{2 \pi}$.

Similarly, the critical pressure can be obtained and is given by

$p_{c}=\frac{1-a}{2 \pi v_{c}^{2}}-\frac{6 Q^{2}}{\pi v_{c}^{4}}$.

From the condition given by Eq. (40), we get the critical values of the thermodynamics quantities, namely,

$v_{c}=\frac{2 \sqrt{6} Q}{1-a}, \quad T_{c}=\frac{(1-a)^{2}}{2 \sqrt{\pi} Q}\left(\frac{2-a}{3}\right)-\frac{\alpha}{2 \pi}$,

$p_{c}=\frac{(1-a)^{3}(1+a)}{96 \pi Q^{2}}$.

The combined effects of the cloud of strings and quintessence are shown explicitly in Eq. (43). Note that if we consider $a=0$, which means that there is no cloud of strings, we get the results already obtained in the literature [36], when the Reissner-Nordstrom AdS black hole surrounded by quintessence was considered. Similarly, in the absence of the quintessence and the cloud of strings, we get the known critical variables of the static charged black hole in a cosmological constant background [17]. The values of the critical thermodynamic quantities, when we vary the string cloud parameter $a$, are represented in Tables 1, 2 and 3. The diagram $p-V$ represented in Fig. 6 shows the critical behavior for different values of the parameter $a$. In this case, the critical value of the temperature, $T_{c}$, is 0.14472 .

Finally, the Gibbs free energy of the black hole is defined by

$G(T, Q, p)=M(S, Q, p)-T S=\frac{1-a}{4} r_{h}+\frac{3 Q^{2}}{4 r_{h}}-\frac{2 \pi p r_{h}^{3}}{3}$,

and its behavior is represented in the Fig. 8 .

Table 1 Behaviour of the critical parameters $v_{c}, T_{c}, p_{c}$ and $\frac{p_{c} v_{c}}{T_{c}}$ for different values of $a$ and for $Q=1, \alpha=0.01$ and $\omega_{q}=-2 / 3$

\begin{tabular}{lllll}
\hline$a$ & $v_{c}$ & $T_{c}$ & $p_{c}$ & $\frac{p_{c} v_{c}}{T_{c}}$ \\
\hline 0 & 4.89898 & 0.18647 & 0.00332 & 0.08711 \\
0.05 & 5.15682 & 0.16389 & 0.00298 & 0.09392 \\
0.1 & 5.44331 & 0.14312 & 0.00266 & 0.10112 \\
0.15 & 5.76350 & 0.12409 & 0.00234 & 0.10876 \\
0.2 & 6.12372 & 0.10673 & 0.00203 & 0.11688 \\
0.25 & 6.53197 & 0.0097 & 0.00175 & 0.12555
\end{tabular}

Table 2 Behaviour of the critical parameters $v_{c}, T_{c}, p_{c}$ e $\frac{p_{c} v_{c}}{T_{c}}$ for different values of $\alpha$ and for $Q=1, a=0.1$ and $\omega_{q}=-2 / 3$

\begin{tabular}{lllll}
\hline$\alpha$ & $v_{c}$ & $T_{c}$ & $p_{c}$ & $\frac{p_{c} v_{c}}{T_{c}}$ \\
\hline 0.01 & 5.44331 & 0.14312 & 0.00266 & 0.10112 \\
0.05 & 5.44331 & 0.13676 & 0.00266 & 0.10583 \\
0.1 & 5.44331 & 0.12880 & 0.00266 & 0.11237 \\
0.2 & 5.44331 & 0.11288 & 0.00266 & 0.12821 \\
0.3 & 5.44331 & 0.09697 & 0.00266 & 0.14926 \\
\hline
\end{tabular}

Table 3 Behaviour of the critical parameters $v_{c}, T_{c}, p_{c}$ e $\frac{p_{c} v_{c}}{T_{c}}$ for different values of $\alpha$ and for $Q=1, a=0.25$ and $\omega_{q}=-2 / 3$

\begin{tabular}{lllll}
\hline$\alpha$ & $v_{c}$ & $T_{c}$ & $p_{c}$ & $\frac{p_{c} v_{c}}{T_{c}}$ \\
\hline 0.01 & 6.53197 & 0.09097 & 0.00154 & 0.11048 \\
0.05 & 6.53197 & 0.08461 & 0.00154 & 0.11880 \\
0.1 & 6.53197 & 0.07665 & 0.00154 & 0.13113 \\
0.2 & 6.53197 & 0.06073 & 0.00154 & 0.16550 \\
0.3 & 6.53197 & 0.04482 & 0.00154 & 0.22427 \\
\hline
\end{tabular}
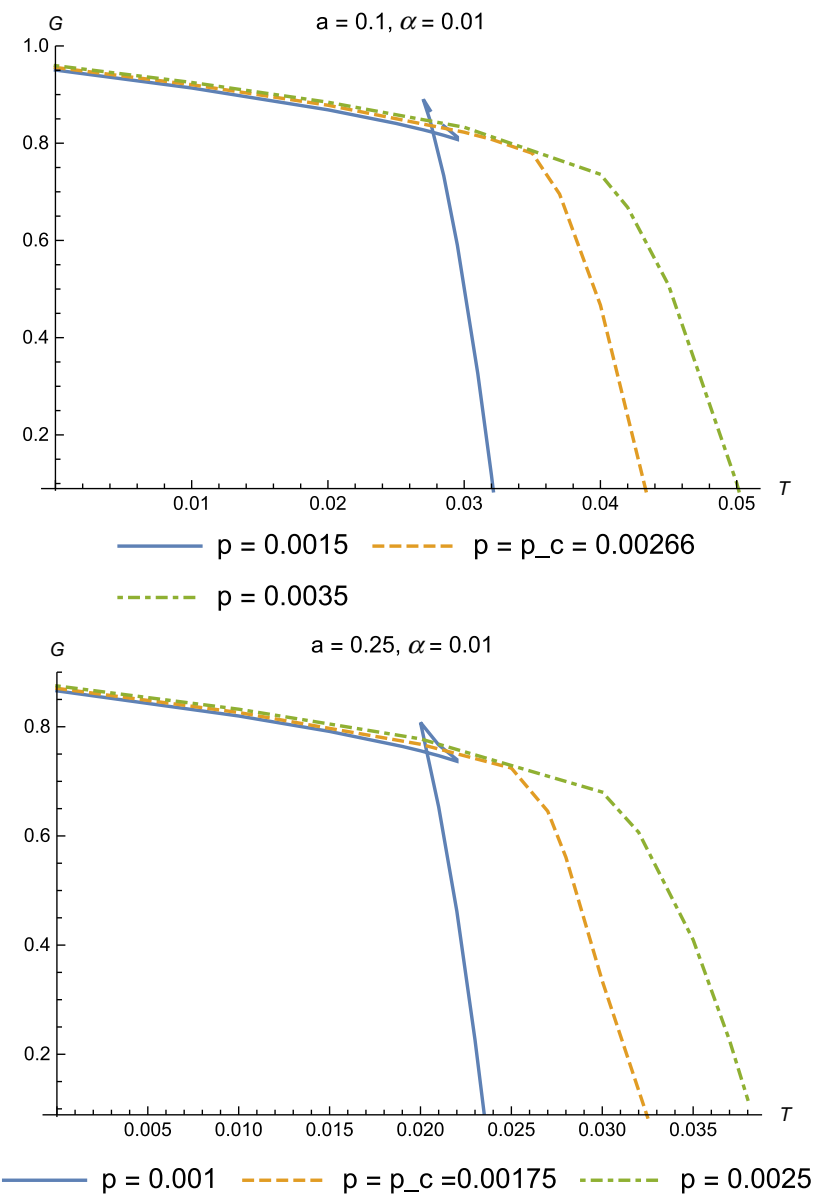

Fig. 8 Graph of the Gibbs free energy as a function of the Hawking temperature $T$,for $Q=1$ 
The critical exponents help us to describe the behavior of a system near the critical point [36]. To this proposal, let us define, for convenience, the following quantities

$t=\frac{T}{T_{c}}-1, \quad \epsilon=\frac{v}{v_{c}}-1, \quad \tilde{p}=\frac{p}{p_{c}}$.

Thus, the critical exponents can be expressed in terms of a power law, as [36]:

$C_{v}=\propto|t|^{-\xi}$

$\eta \propto|t|^{\beta}$

$\kappa_{T} \propto|t|^{-\gamma}$

$\left|p-p_{c}\right| \propto\left|v-v_{c}\right|^{\delta}$,

where $\xi, \beta, \gamma$ and $\delta$ characterize the behavior of the heat capacity at constant volume, order parameter, isothermal compressibility, and pressure, respectively.

According to Eq. (31), $C_{V}=0$ and, thus, $\xi=0$. Now, let us consider the behavior of the equation of state in the neighborhood of the critical point. Thus, Eq. (38) can be expanded as [36]

$\tilde{p}=1+b_{10} t+b_{01} \epsilon+b_{11} t \epsilon+b_{02} \epsilon^{2}+b_{03} \epsilon^{3}+\mathscr{O}\left(t \epsilon^{2}, \epsilon^{4}\right)$,

where

$b_{10}=b_{02}=0$,

$b_{10}=\frac{T_{c}}{p_{c} v_{c}}$

$b_{11}=-\frac{T_{c}}{p_{c} v_{c}}$,

$b_{03}=\frac{24 T_{c}}{p_{c} v_{c}}-\frac{18(1-a)}{\pi p_{c} v_{c}^{2}}+\frac{12 \alpha}{p_{c} v_{c}}$.

Assuming that the black hole undergoes a transition from a large state, $l$, for a small one, $s$, and that, during the phase transition, the pressure is kept constant, $\mathrm{Li}$ [36] verified that

$\epsilon_{l}=-\epsilon_{s}=\sqrt{\frac{-b_{11} t}{b_{03}}}$

Therefore, the order parameter has the following behavior in terms of the reduced temperature

$\eta=v_{l}-v_{s}=v_{c}\left(\epsilon_{l}-\epsilon_{s}\right)=2 v_{c} \sqrt{\frac{-b_{11} t}{b_{03}}} \propto(-t)^{1 / 2}$

Thus, we get $\beta=1 / 2$. As to the isothermal compressibility, $\kappa_{T}$, we get the following result
$\kappa_{T}=-\left.\frac{1}{v} \frac{\partial v}{\partial p}\right|_{v_{c}} \propto-\left.\frac{1}{\frac{\partial \tilde{p}}{\partial \epsilon}}\right|_{\epsilon=0}=-\frac{1}{b_{11} t}$,

and, as a consequence, we obtain that $\gamma=1$. Finally, if we consider the reduced temperature equal to zero, $t=0$, we get

$\tilde{p}-1=b_{03} \epsilon^{3}$

which gives us that $\delta=3$.

From these results we conclude that the critical exponents are exactly the same obtained for a Reissner-Nordström-AdS black hole surrounded by quintessence [36] Thus, the presence of the cloud of strings does not modify the values of the critical exponents.

\section{Black hole as heat engine}

We will deal with a black hole as a thermo-gravitational heat engine $[26,38]$. As it is known, heat engines are devices that, receiving energy from a hot source $\left(Q_{H}\right)$, are able to do an work $(W)$, transferring the residual heat $\left(Q_{C}\right)$ to a cold reservoir. The efficiency of a heat engine $(\eta)$ is, thus, defined as the ratio between the work it produces and the heat it receives, which is expressed by

$\eta=\frac{W}{Q_{H}}=1-\frac{Q_{C}}{Q_{H}}$.

where we used the energy conservation law $Q_{H}=W+$ $Q_{C}$, with the work done in a cycle, $W$, being given exactly by the area of the cycle in the $p-V$ plane. As a black hole can be considered a heat engine with the thermodynamic potentials determined in the last section, in what follows we will consider that it operates in two different cycles.

Firstly, we consider that the black hole operates in a cycle formed by two isobaric and two isochoric processes, as shown in Fig. 9. This rectangular cycle can be used to built cycles of arbitrary shapes, by using a large number of small rectangular cycles.

We will compare the efficiency of the engine described above with the one of the engine that produces the maximal efficiency. The classical Carnot heat engines, which operates by two isothermal and two adiabatic processes, produces the maximal efficiency, which it is given by

$\eta_{c}=1-\frac{T_{C}}{T_{H}}$

with $T_{C}$ and $T_{H}$ being the temperatures of the cold source and the hot reservoir, respectively. As can be seen from Eq. (28), the thermodynamical volume of the black hole is a function of the entropy [26]. Thus, for the system considered, the 


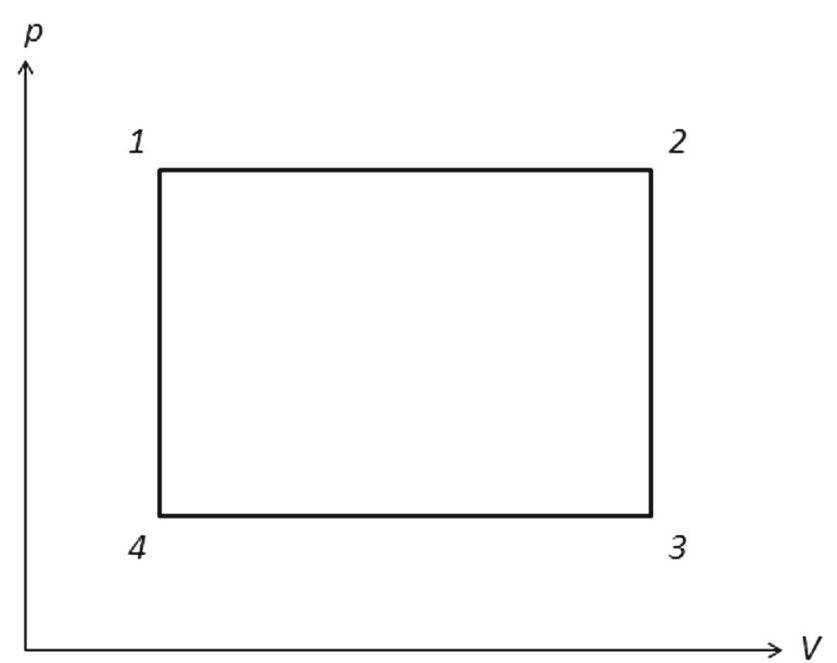

Fig. $9 p \times V$ diagram of a heat engine operating by two isobaric and two isochoric processes

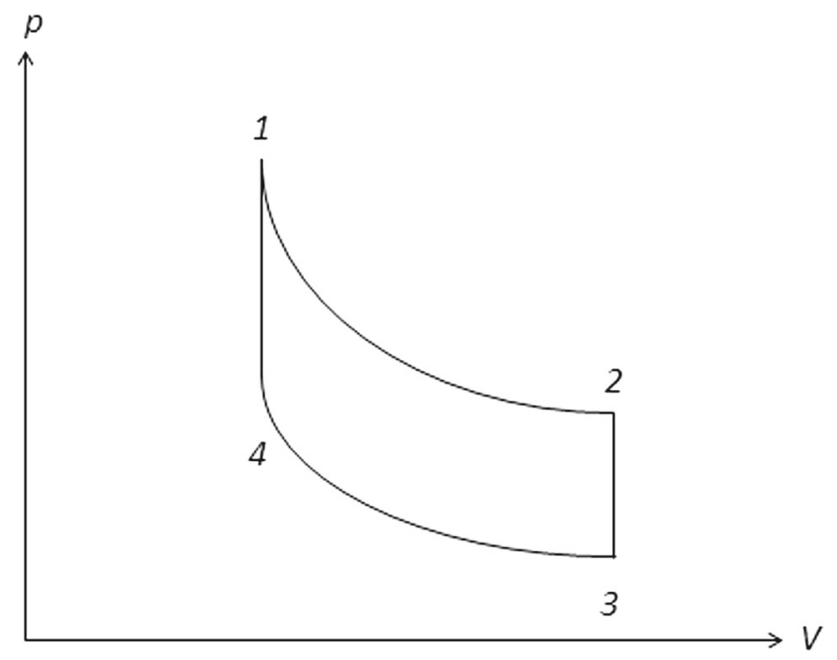

Fig. $10 p \times V$ diagram of a heat engine that produces the maximal efficiency

adiabatic processes ( $S$ constant) are also isochoric and the cycle represented in Fig. 10 (in which $1 \rightarrow 2$ and $3 \rightarrow 4$ are isothermic processes) will produce the maximal efficiency.

In the cycle represented in Fig. 9, which is calculated by the area of the figure formed, we obtain that

$W=\left(V_{2}-V_{1}\right)\left(p_{1}-p_{4}\right)=\frac{4}{3 \sqrt{\pi}}\left(S_{2}^{3 / 2}-S_{1}^{3 / 2}\right)\left(p_{1}-p_{4}\right)$.

As discussed above, there is no heat exchange in isochoric processes and $Q_{H}$ is the heat received in the isobaric process $1 \rightarrow 2$. Thus, from Eq. (30), we obtain

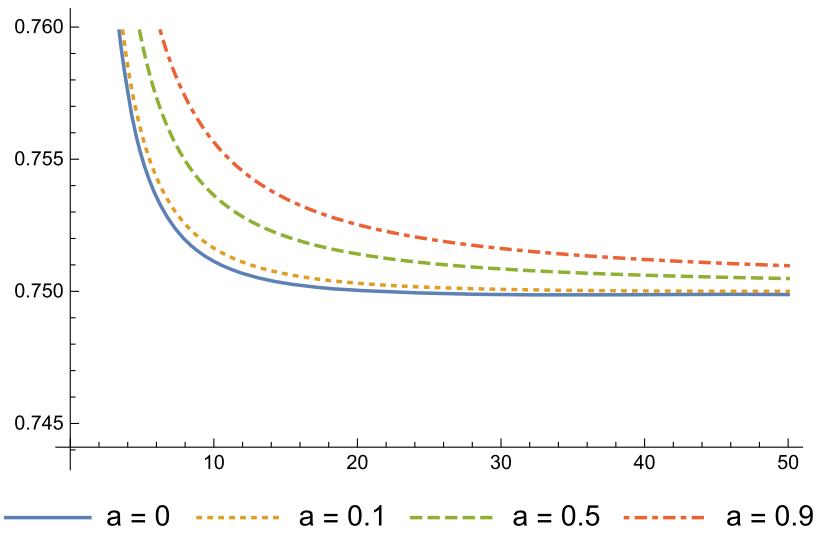

Fig. 11 Parameter, $\eta$, as a function of the entropy, $S$, for different values of $a$

$$
\begin{aligned}
Q_{H}= & \int_{T_{1}}^{T_{2}} C_{p}\left(p_{1}, T\right) d T=\left.\int_{S_{1}}^{S_{2}} C_{p}\left(p_{1}, S\right) \frac{\partial T}{\partial S}\right|_{p} d S \\
= & \int_{S_{1}}^{S_{2}} T d S=\int_{1}^{2} d M=M_{2}-M_{1} \\
= & \frac{1-a}{2} \sqrt{\frac{S_{2}}{\pi}}+\frac{Q^{2}}{2} \sqrt{\frac{\pi}{S_{2}}}-\frac{\alpha}{2} \frac{S_{2}}{\pi}+\frac{4 \pi p_{2}}{3}\left(\frac{S_{2}}{\pi}\right)^{3 / 2} \\
& -\left[\frac{1-a}{2} \sqrt{\frac{S_{1}}{\pi}}+\frac{Q^{2}}{2} \sqrt{\frac{\pi}{S_{1}}}-\frac{\alpha}{2} \frac{S_{1}}{\pi}+\frac{4 \pi p_{1}}{3}\left(\frac{S_{1}}{\pi}\right)^{3 / 2}\right] .
\end{aligned}
$$

Therefore, the efficiency of the thermogravitational machine is given by

$\eta=\frac{\left(1-\frac{p_{4}}{p_{1}}\right)}{\xi}$

where

$$
\begin{aligned}
\xi= & +\frac{3(1-a)\left(\sqrt{S_{2}}-\sqrt{S_{1}}\right)}{8\left(S_{2}^{3 / 2}-S_{1}^{3 / 2}\right)} \\
& +\frac{3 \pi Q^{2}}{8\left(S_{2}^{3 / 2}-S_{1}^{3 / 2}\right)}\left(\frac{1}{\sqrt{S_{2}}}-\frac{1}{\sqrt{S_{2}}}\right) \\
& -\frac{3 \alpha}{8 \sqrt{\pi}} \frac{S_{2}-S_{1}}{S_{2}^{3 / 2}-S_{1}^{3 / 2}} .
\end{aligned}
$$

Otherwise, the efficiency of the Carnot engine is calculated by [26]:

$\eta_{c}=1-\frac{T_{4}\left(p_{4}, S_{1}\right)}{T_{2}\left(p_{1}, S_{2}\right)}$

In Figs. 11 and 12, we represent the behaviour of $\eta$ and of the ratio $\eta / \eta_{c}$ for different values of the cloud of string parameter $a$.

Note that, when the parameter $a$ increases, the efficiency increases as shown in Fig. 11. On the other hand, Fig. 12 show 


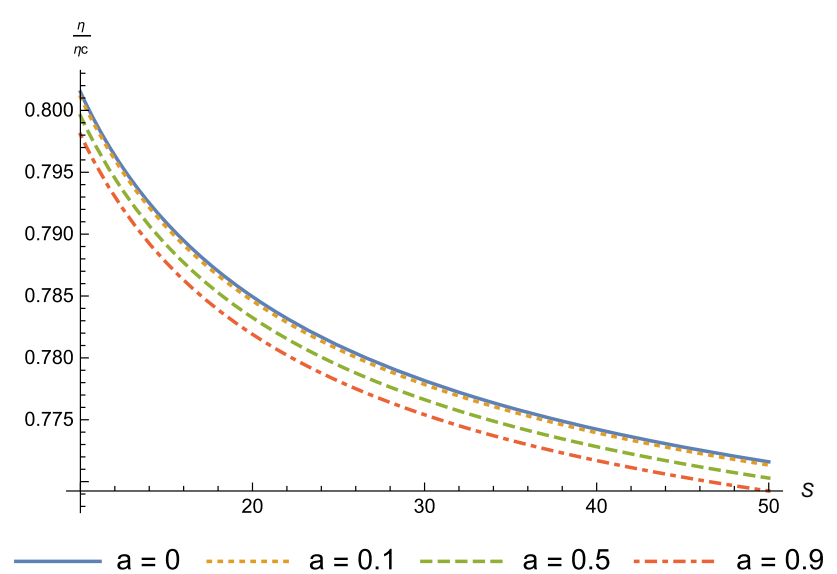

Fig. 12 Rate of $\eta / \eta_{c}$ as a function of the entropy, $S$, for different values of $a$

us that, when $a$ increases, the ratio between the efficiency of the engine and the maximal efficiency decreases.

\section{Concluding remarks}

We considered the cosmological constant as the thermodynamic pressure and analyzed the thermodynamics of the Reissner-Nordstrom AdS black hole surrounded by a cloud of strings and quintessence, for the specific case with $\omega_{q}=$ $-2 / 3$, and obtained analytic expressions for the thermodynamical quantities as well as for their values in the critical points. The thermodynamic quantities as temperature, entropy, internal energy, and heat capacity are analogous to the corresponding expressions when only the quintessence is present [36]. In fact, the role played by the cloud of strings is to decrease these quantities as compared to both cases, namely, when only the quintessence is present.

These effects due to the cloud of strings are shown in Fig. 2, for the mass parameter, and in Fig 3, for the Hawking temperature. Otherwise, the analytic expression corresponding to the internal energy, given by Eq. (29), and the one corresponding to the heat capacity at constant pressure, given by Eq. (30), show us that the effect of the cloud of strings is to decrease the values of these quantities as compared with the case in which only the quintessence is present [36].

In which concerns the critical Hawking temperature, critical pressure and critical specific volume, given by Eqs. (41), (42) and (43), respectively, they are affected by the presence of the cloud of strings, which decreases the values of these quantities when compared with the case where only the quintessence is present. Note that, when the cloud of strings is absent, the results coincide with the ones already obtained in the literature [36]. It is worth calling attention to the fact that the critical specific volume, $v_{c}$, increases when the parameter which codifies the presence of the cloud of strings, $a$, increases, as shown in Table 1 . On the other hand, when $a$ increases, the critical temperature, $T_{c}$, and the critical pressure, $p_{c}$, decreases, as shown in Table 1 .

When we consider the effect of the normalization factor, as shown in Tables 2 and 3 , we conclude that in the presence of the cloud of strings, for the values assumed for this factor, the critical specific volume and the critical pressure are, approximately, constants, while the critical temperature varies. Therefore, the ration $p_{c} v_{c} / T_{c}$ is not constant, but depends on the normalization factor, $\alpha$, as well as on the parameter $\omega_{q}$, which, in this paper, we fixed such that $\omega_{q}=-2 / 3$. It is worth calling attention to the fact that the critical exponents have the same values as the ones obtained when only the quintessence is present [36], which means that the presence of the cloud of strings does not affect these parameters.

As to the heat engine, we investigated the effects of the cloud of strings, for fixed $\omega_{q}$, on its efficiency. The efficiency, as shown in Fig. 11, increases when the parameter $a$ increases, for fixed value of $\omega_{q}$. Thus, the presence of the cloud of strings improves the heat engine efficiency which will increases as the parameter $a$ grows. On the other hand, the ratio $\eta / \eta_{c}$ decreases with the parameter $a$ which codifies the presence of the cloud of strings.

The results obtained, in fact, show us how the thermodynamics of the system under consideration is affected by the presence of the cloud of strings as well as by the quintessence.

Acknowledgements V. B. Bezerra is partially supported by Conselho Nacional de Desenvolvimento Científico e Tecnológico (CNPq) through the research Project no. 305835/2016-5.

Data Availability Statement This manuscript has no associated data or the data will not be deposited. [Authors' comment: Our paper concerns only to theoretical investigations and thus it does not contain any associated data.]

Open Access This article is distributed under the terms of the Creative Commons Attribution 4.0 International License (http://creativecomm ons.org/licenses/by/4.0/), which permits unrestricted use, distribution, and reproduction in any medium, provided you give appropriate credit to the original author(s) and the source, provide a link to the Creative Commons license, and indicate if changes were made. Funded by SCOAP ${ }^{3}$.

\section{References}

1. P.S. Letelier, Phys. Rev. D 20(6), 1294 (1979)

2. M. Barriola, A. Vilenkin, Phys. Rev. Lett. 63(4), 341 (1989)

3. S. Perlmutter, G. Aldering, G. Goldhaber, R. Knop, P. Nugent, P. Castro, S. Deustua, S. Fabbro, A. Goobar, D.E. Groom et al., Astrophys. J. 517(2), 565 (1999)

4. V.V. Kiselev, Class. Quantum Grav. 20(6), 1187 (2003)

5. J.D. Bekenstein, Lett. Nuovo Cimento (1971-1985) 4(15), 737 (1972)

6. J.D. Bekenstein, Phys. Rev. D 7(8), 2333 (1973)

7. S.W. Hawking, Nature 248(5443), 30 (1974) 
8. S.W. Hawking, Comm. Math. Phys. 43(3), 199 (1975)

9. S.W. Hawking, Phys. Rev. D 13(2), 191 (1976)

10. A. Chamblin, R. Emparan, C.V. Johnson, R.C. Myers, Phys. Rev. D 60(6), 064018 (1999)

11. A. Chamblin, R. Emparan, C.V. Johnson, R.C. Myers, Phys. Rev. D 60(10), 104026 (1999)

12. M.M. Caldarelli, G. Cognola, D. Klemm, Class. Quantum Grav. 17(2), 399 (2000)

13. W. Shuang, W. Shuang-Qing, X. Fei, D. Lin, Chin. Phys. Lett. 23(5), 1096 (2006)

14. Y. Sekiwa, Phys. Rev. D 73(8), 084009 (2006)

15. D. Kastor, S. Ray, J. Traschen, Class. Quantum Grav. 26(19), $195011(2009)$

16. B.P. Dolan, Class. Quantum Grav. 28(12), 125020 (2011)

17. D. Kubiznák, R.B. Mann, J. High Energy Phys. 2012(7), 33 (2012)

18. R.G. Cai, L.M. Cao, L. Li, R.Q. Yang, J. High Energy Phys. 2013(9), 5 (2013)

19. R. Banerjee, D. Roychowdhury, Phys. Rev. D 85(10), 104043 (2012)

20. H. Liu, X.H. Meng, Mod. Phys. Lett. A 31(37), 1650199 (2016)

21. D.C. Zou, S.J. Zhang, B. Wang, Phys. Rev. D 89(4), 044002 (2014)

22. B.P. Dolan, Phys. Rev. D 84(12), 127503 (2011)
23. M. Cvetič, G. Gibbons, D. Kubizňák, C. Pope, Phys. Rev. D 84(2), 024037 (2011)

24. E. Spallucci, A. Smailagic, Phys. Lett. B 723(4-5), 436 (2013)

25. S. Hendi, R. Mann, S. Panahiyan, B.E. Panah, Phys. Rev. D 95(2), 021501 (2017)

26. H. Liu, X.H. Meng, Eur. Phys. J. C 77(8), 556 (2017)

27. J.X. Mo, W.B. Liu, Eur. Phys. J. C 74(4), 2836 (2014)

28. R. Zhao, H.H. Zhao, M.S. Ma, L.C. Zhang, Eur. Phys. J. C 73(12), 2645 (2013)

29. C.V. Johnson, Class. Quantum Grav. 31(20), 205002 (2014)

30. J.X. Mo, F. Liang, G.Q. Li, J. High Energy Phys. 2017(3), 10 (2017)

31. M.R. Setare, H. Adami, Gen. Relativ. Grav. 47(11), 133 (2015)

32. C.V. Johnson, Entropy 18(4), 120 (2016)

33. J. Sadeghi, K. Jafarzade. arXiv preprint. arXiv:1504.07744 (2015)

34. C.V. Johnson, Class. Quantum Grav. 33(21), 215009 (2016)

35. J. Toledo, V. Bezerra, Int. J. Mod. Phys. D 28, 1950023 (2019)

36. G.Q. Li, Phys. Lett. B 735, 256 (2014)

37. W. Xu, Y. Wu, EPL (Europhys. Lett.) 121(4), 40001 (2018)

38. R.A. Hennigar, F. McCarthy, A. Ballon, R.B. Mann, Class. Quantum Grav. 34(17), 175005 (2017) 